\title{
Full-field predictions of ice dynamic recrystallisation under simple shear conditions
}

\author{
Maria-Gema Llorens ${ }^{\mathrm{a}, \mathrm{b}, *}$, Albert Griera ${ }^{\mathrm{c}}$, Paul D. Bons ${ }^{\mathrm{a}}$, Ricardo A. Lebensohn ${ }^{\mathrm{d}}$, \\ Lynn A. Evans ${ }^{e}$, Daniela Jansen ${ }^{\mathrm{b}}$, Ilka Weikusat ${ }^{\mathrm{a}, \mathrm{b}}$ \\ a Department of Geosciences, Eberhard Karls University Tübingen, Germany \\ ${ }^{\mathrm{b}}$ Alfred Wegener Institute for Polar and Marine Research, Bremerhaven, Germany \\ c Departament de Geologia, Universitat Autònoma de Barcelona, Spain \\ ${ }^{\mathrm{d}}$ Material Science and Technology Division, Los Alamos National Laboratory, USA \\ e School of Earth, Atmosphere and Environmental Sciences, Monash University, Clayton, Victoria, Australia
}

\section{A R T I C L E I N F O}

\section{Article history:}

Received 14 January 2016

Received in revised form 22 June 2016

Accepted 23 June 2016

Available online $\mathrm{xxxx}$

Editor: A. Yin

\section{Keywords:}

dynamic recrystallisation

ice microstructure

strain localisation

viscoplastic anisotropy

\begin{abstract}
A B S T R A C T
Understanding the flow of ice on the microstructural scale is essential for improving our knowledge of large-scale ice dynamics, and thus our ability to predict future changes of ice sheets. Polar ice behaves anisotropically during flow, which can lead to strain localisation. In order to study how dynamic recrystallisation affects to strain localisation in deep levels of polar ice sheets, we present a series of numerical simulations of ice polycrystals deformed under simple-shear conditions. The models explicitly simulate the evolution of microstructures using a full-field approach, based on the coupling of a viscoplastic deformation code (VPFFT) with dynamic recrystallisation codes. The simulations provide new insights into the distribution of stress, strain rate and lattice orientation fields with progressive strain, up to a shear strain of three. Our simulations show how the recrystallisation processes have a strong influence on the resulting microstructure (grain size and shape), while the development of lattice preferred orientations (LPO) appears to be less affected. Activation of non-basal slip systems is enhanced by recrystallisation and induces a strain hardening behaviour up to the onset of strain localisation and strain weakening behaviour. Simulations demonstrate that the strong intrinsic anisotropy of ice crystals is transferred to the polycrystalline scale and results in the development of strain localisation bands than can be masked by grain boundary migration. Therefore, the finite-strain history is non-directly reflected by the final microstructure. Masked strain localisation can be recognised in ice cores, such as the EDML, from the presence of stepped boundaries, microshear and grains with zig-zag geometries.
\end{abstract}

(C) 2016 Elsevier B.V. All rights reserved.

\section{Introduction}

There is an increasing scientific interest in polar ice sheets because of the role they play in controlling sea-level rise and paleoclimate records through deep drilling projects (e.g. NEEM, 2013). For a correct interpretation of these records, it is crucial to determine to what extent deformation processes, such as dynamic recrystallisation and heterogeneous flow, may affect or distort ice stratigraphy, especially in the oldest and, thus, deepest ice, which can undergo strong deformation (Cuffey and Paterson, 2010; Montagnat et al., 2014).

\footnotetext{
* Corresponding author at: Department of Geosciences, Eberhard Karls University Tübingen, Germany.

E-mail address: maria-gema.Ilorens-verde@uni-tuebingen.de (M.-G. Llorens).
}

On Earth surface, the only stable ice phase is the hexagonal symmetry form $I h$, which exhibits highly anisotropic viscoplastic deformation. The orientation of ice crystals is usually defined by the orientation of their c-axis, which is perpendicular to the basal plane. Deformation is mostly accommodated by dislocation glide on that plane, while the other slip systems (prismatic and pyramidal) are much harder to activate (e.g. Duval and Castelnau, 1995). A parameter accounting for the anisotropy of the crystal $(A)$ can be defined as the ratio of critical resolved shear stress (CRSS) required to activate slip along non-basal versus basal systems. Experiments reviewed in Duval et al. (1983) indicate that the activation of nonbasal systems requires stresses at least 60 times greater those on basal planes.

Polycrystalline ice with randomly orientated c-axes is considered to behave isotropically, as enough basal planes well oriented for slip are available. However, ice core analysis reveals that caxes tend to rotate towards the vertical direction below a certain 
depth (S.H. Faria et al., 2014). Consequently, ice crystals tend to be oriented with their basal planes close to perpendicular to the maximum compression or shortening direction (e.g. Alley, 1988; Montagnat et al., 2014). Ice polycrystals in ice sheets and glaciers thus form a lattice preferred orientation (LPO) and develop a bulk intrinsic anisotropy with deformation (S.H. Faria et al., 2014). Near the ice-sheet base, simple shear deformation dominates and a strong LPO develops (S.H. Faria et al., 2014). It has been found that ice with a strong LPO deforms up to an order of magnitude faster than isotropic ice, and therefore, ice flow strongly depends on the LPO of the polycrystalline ice (Azuma, 1994; Castelnau et al., 1996).

Ice microstructure is affected by different recrystallisation processes, depending on the temperature and strain rate (H.G. Faria et al., 2014). Dynamic recrystallisation affects the evolution of the microstructure (grain-size and shape) and influences the development of LPOs (e.g. Montagnat et al., 2015). This process is very efficient in ice due to the natural deformation conditions of this mineral, i.e. temperatures close to its pressure melting point and deformation at low strain rates (Kipfstuhl et al., 2006). These are observable especially in comparison with fast artificial deformation in creep tests (Weikusat et al., 2009).

Near the flow divide of ice sheets, ice deforms under vertical compression and horizontal extension (Montagnat et al., 2014). On the contrary, simple shear boundary conditions dominate in zones away from the divide and close to the bedrock (e.g. Cuffey and Paterson, 2010). It is therefore crucial for understanding bulk polar ice behaviour to study the fabrics and microstructural evolution of polar ice in response to stress and time under different types of deformation (i.e. under different strain rates and ratio between vertical and shear strain rates or kinematic vorticity).

New insights into ice rheology can be gained from interpretations of deformed natural ice from ice cores (S.H. Faria et al., 2014) or from experiments of natural or artificial ice deformed in the laboratory (Azuma, 1994; Treverrow et al., 2012; Montagnat et al., 2015). However, ice experiments in the laboratory are performed at high strain rates compared with that in natural conditions (Budd and Jacka, 1989) and high finite strains cannot be achieved. Numerical simulations can complement the study of ice rheology, as they can be performed at different strain rates and up to high strain. The aim of this contribution is to systematically study the effects of dynamic recrystallisation on the mechanical behaviour, resulting microstructures and strain localisation of polar ice under simple shear deformation, by means of numerical simulations. We focus on the evolution of fabrics and microstructures in response to finite strain and time, and the associated development of intrinsic polycrystalline anisotropy. We specifically capture the strain localisation indicators in the developed microstructures, and discuss how the resulting finite strain localisation that can potentially affect the spreading of climate signals at the small scale (Faria et al., 2010).

\section{Numerical procedure}

We use a two-dimensional numerical model (software platform ELLE, Bons et al., 2008; http://www.elle.ws) that simulates viscoplastic deformation coupled with dynamic recrystallisation $(D R X)$, in order to investigate the microstructural evolution of an aggregate of pure ice grains. The numerical approach is based on the coupling of a full-field viscoplastic code using the Fast Fourier Transform algorithm (VPFFT; Lebensohn, 2001; Lebensohn et al., 2008) and several ELLE modules (Bons et al., 2008) that simulate recrystallisation processes such as recovery and grain boundary migration (Llorens et al., 2016). The VPFFT formulation provides a solution of the micromechanical problem by finding a strain rate and stress field that minimises the average local work-rate un-
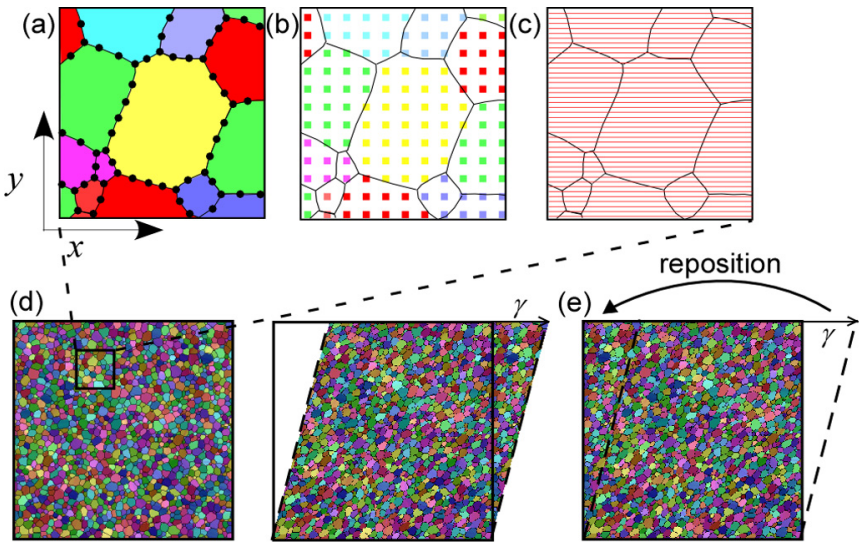

Fig. 1. Data structure. ELLE microstructures consist of three different layers: (a) boundary nodes (triple or double nodes) that define polygons (grains), (b) a regular mesh of unconnected nodes used for the VPFFT calculation, and (c) a passive marker grid used to track the finite deformation, where only initially horizontal grid lines are shown. The initially unit length model (d) is repositioned after the deformation step, bringing the model back into the unit cell (e).

der the compatibility and equilibrium constraints (see Lebensohn, 2001). The algorithm assumes deformation by dislocation glide along pre-defined slip systems. Here we use the crystallography (unit cell dimensions and symmetry) of ice Ih and consider slip of dislocations along the basal, prismatic and pyramidal planes. The deformation-induced lattice rotation and the estimation of geometrically necessary dislocation densities calculated from the stress and velocity fields provided by the VPFFT algorithm are used to simulate intra-crystalline recovery and grain boundary migration. The ELLE platform has previously been used to simulate several deformation microstructure processes, including grain growth (Roessiger et al., 2011), dynamic recrystallisation (Piazolo et al., 2002), strain localisation (Griera et al., 2011; 2013) or folding (Llorens et al., 2013a; 2013b). In this study, grain boundary migration and recovery processes are used in order to simulate the microstructural evolution by recrystallisation. Grain boundary migration $(G B M)$ is simulated using a front-tracking approach based on the algorithm by Becker et al. (2008). Grain boundary migration is driven by reduction of grain-boundary energy and stored strain energy, reproducing the motion or displacement of highangle grain boundaries (HAGB). Regions swept by grain boundaries are assumed to restore lattice distortions (i.e. dislocation densities are zero). Recovery reduces the intra-granular stored energy in a deformed crystal by the annihilation of dislocations and their rearrangement into low-angle subgrains (Borthwick et al., 2013). A limitation of the model is that nucleation of new HAGB from lobate boundaries or due to highly deformed grains is still not implemented.

The data structure of the models consists of two basic layers (Fig. 1): (a) a contiguous set of polygons (termed flynns) that are themselves defined by boundary nodes (bnodes) and are connected by straight boundary segments, and (b) a set of unconnected nodes (unodes) that provide a high-resolution grid for storing physical properties within grains. Additionally, an extra layer of unconnected nodes is used as a passive marker grid to track the finite deformation (Fig. 1c). A resolution of $256 \times 256$ Fourier points (unodes) was used to map lattice orientations, resulting in a unit cell defined by 65,536 discrete unodes. Each unode represents a small area with a certain lattice orientation, defined by three Euler angles following the Bunge convention, and local parameters, such as stress or dislocation density. The VPFFT code uses these unodes for the viscoplastic deformation calculations. The change of the position of bnodes is calculated according to the deformation velocity field and grain boundary migration. The data structure of both the 

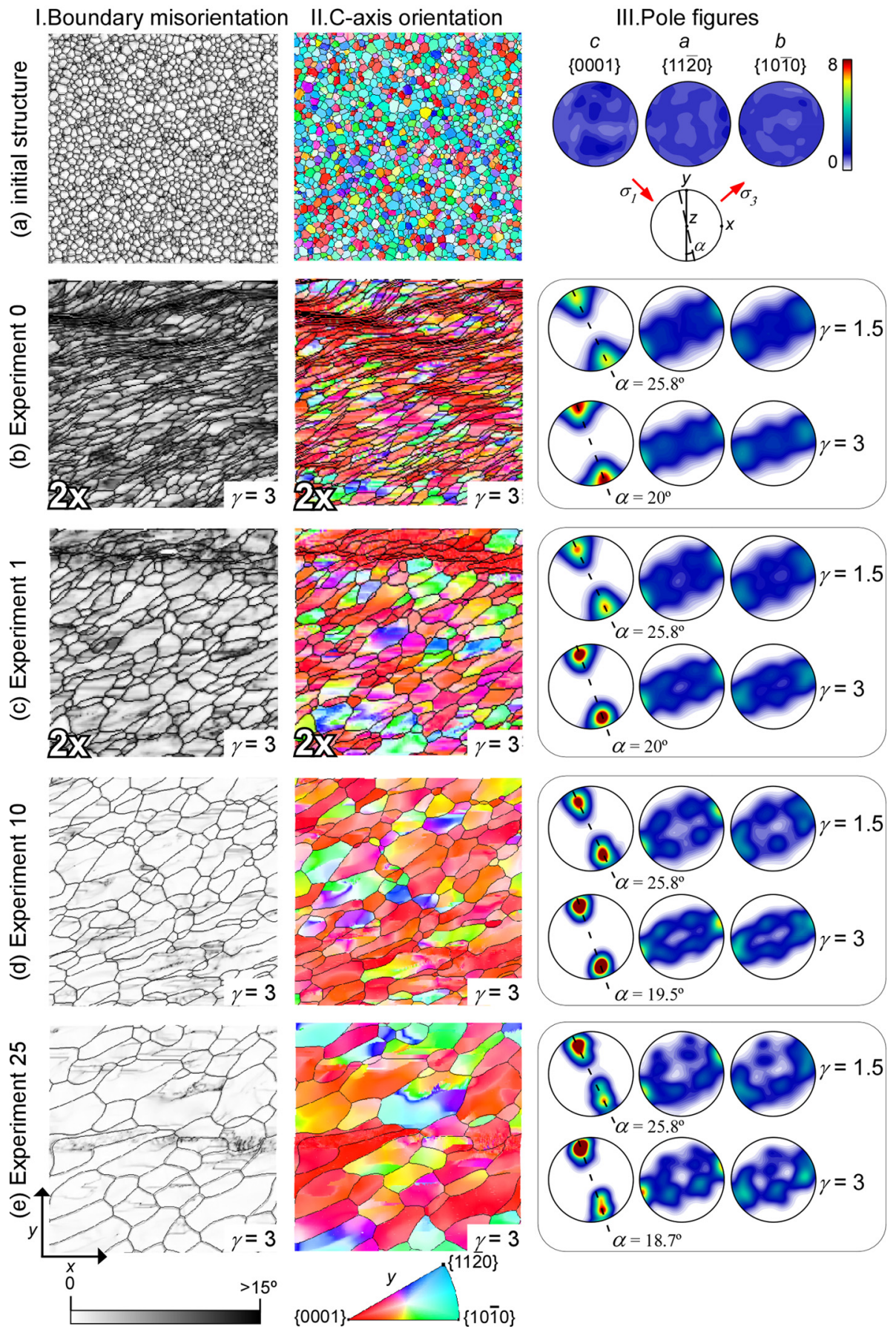

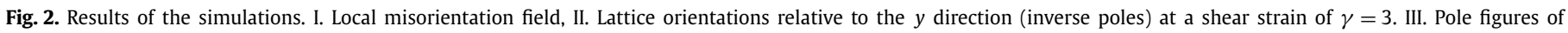

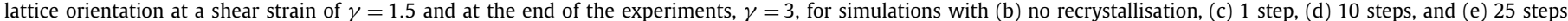

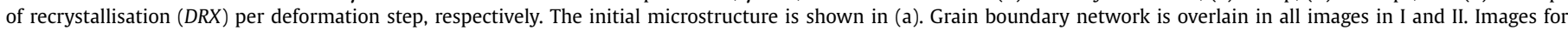

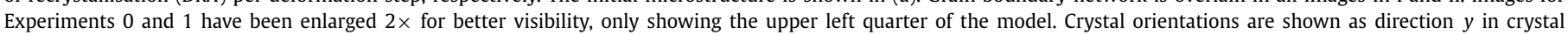
reference frame (IPF). Colour bar indicates the multiples of uniform distribution.

VPFFT and ELLE codes are fully wrapping: grains touching one side of the model continue on the other side. This feature not only reduces boundary effects, but also allows repositioning the model into a square grid every time step, and therefore, large strains can be reached (Fig. 1e).

Each process in ELLE is performed sequentially in a loop that represents a small time increment ( $\Delta t$; Bons et al., 2008). Each loop starts with the application of a viscoplastic (VPFFT) dextral shear strain increment of $\Delta \gamma=0.04$. After this deformation step, the microstructure is subjected to a sub-loop of dynamic recrys- tallisation processes $(D R X)$ : grain boundary migration and recovery. As the mobility and time step for the DRX processes is the same for all the simulations, this sub-loop may be called more than once to simulate deformation at different strain rates, varying the balance between deformation and recrystallisation (see Llorens et al., 2016).

The input microstructure has 1632 grains and a size of $10 \times$ $10 \mathrm{~cm}^{2}$. Grains have an initially random lattice orientation (Fig. 2a columns II and III), representing a bulk isotropic material. The ratio of critical resolved shear stress for non-basal versus basal slip sys- 
Table 1

Experimental setup.

\begin{tabular}{|c|c|c|c|c|c|}
\hline Experiment name & $\begin{array}{l}D R X / F F T \\
\text { ratio }\end{array}$ & $\begin{array}{l}\text { Initial average grain area } \\
\left(\mathrm{mm}^{2}\right)\end{array}$ & $\begin{array}{l}\text { Final average grain area } \\
\left(\mathrm{mm}^{2}\right)\end{array}$ & $\begin{array}{l}\text { Final number } \\
\text { of grains }\end{array}$ & $\begin{array}{l}\text { Strain rate } \\
\left(\mathrm{s}^{-1}\right)\end{array}$ \\
\hline Experiment 0 & 0 & 6.12 & 6 & 1636 & - \\
\hline Experiment 1 & 1 & 6.12 & 12 & 873 & $3.17 \times 10^{-11}$ \\
\hline Experiment 10 & 10 & 6.12 & 64 & 156 & $3.17 \times 10^{-12}$ \\
\hline Experiment 25 & 25 & 6.12 & 163 & 61 & $1.27 \times 10^{-12}$ \\
\hline
\end{tabular}

tems was set to $A=20$ and a value of stress exponent $n=3$ was chosen for all slip systems (e.g. Castelnau et al., 1996). The value of $A$ is assumed to range between 60 and 100 for hexagonal ice $(I h)$. However, previous tests showed that the effect of increasing $A$ is not clearly observable with our approach at values of $A$ above 10 , but significantly increases calculation time (Llorens et al., 2016).

The initial microstructure was deformed up to shear strain of $\gamma=3$ in simple shear. Four simulations at different strain rates were performed by varying the number of steps in the recrystallisation loop (Table 1). Grain boundary mobility (intrinsic mobility $M_{0}=0.23 \mathrm{~m}^{2} \mathrm{~kg}^{-1} \mathrm{~s}^{-1}$; Llorens et al., 2016) and time step ( $d t=20$ years) were the same for all the simulations, and therefore different ratios between the number of $D R X$ and VPFFT steps effectively represent different strain rates.

\subsection{Data analysis and visualisation}

Each simulation produces a wealth of information and data that was analysed and visualised in various ways. The microstructure is visualised in two ways. We show the lattice orientation map (Fig. 2 column II), where each unode is colour coded according to the orientation of its lattice orientation relative to the vertical $y$-axis. These images are comparable with those derived from EBSD analyses (Mainprice et al., 2011). Additionally, we use misorientation maps, where the Kernel Average Misorientation (KAM) is plotted for each unode in the model (Fig. 2 column I). The KAM is defined as the average misorientation angle of a given unode with all of his neighbour unodes.

The LPO is visualised in pole figures using all unodes (Fig. 2 column III). The LPO symmetry or fabric type (Fig. 3) denotes the relative proportion of point (or single maximum, P), girdle $(G)$ and random $(R)$ components of the c-axes distribution. These components were calculated from the three eigenvalues $\left(a_{1}^{(2)}, a_{2}^{(2)}\right.$, $a_{3}^{(2)}$ ) of each pole figure as $\mathrm{P}=a_{1}^{(2)}-a_{2}^{(2)}, \mathrm{G}=2 a_{2}^{(2)}-2 a_{3}^{(2)}$, and $\mathrm{R}=3 a_{3}^{(2)}$ (Vollmer, 1990). Eigenvalues were calculated according to Woodcock (1977) (see Llorens et al., 2016). Lattice orientation data, as well as misorientation maps, were plotted using the texture analysis software MTEX (Mainprice et al., 2011) based on the orientation distribution function (ODF).

Although the applied bulk strain rate is constant at $\Delta \gamma=0.04$ per VPFFT step, the strain rate can vary strongly within the model. To visualise the strain-rate intensity (Fig. 4 columns I and II), we plotted the Von Mises strain rate (or equivalent strain rate) for each unode, which is the second invariant of the symmetric strain rate tensor. To determine the finite-strain field, the position of material points or "particles" that were initially arranged on an orthogonal grid were tracked during deformation. The finite deformation was visualised by drawing lines that connect particles that originally formed horizontal lines (Figs. 1c and 4 columns III and IV).

Finally, as the FFT-algorithm calculates the stress state for each unode, the bulk stress can be calculated by averaging all the stresses of the individual unodes. The FFT-algorithm does not use absolute stresses, but normalised stresses to the stress required to activate the basal slip system. The equivalent stress from numerical simulations was scaled using the compilation of experimental

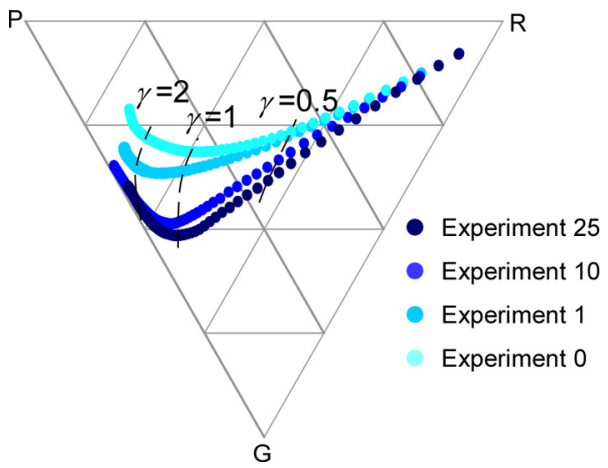

Fig. 3. Ice LPO symmetry for all the experiments performed in this study, expressed as the proportion of point $(P)$, girdle $(G)$ and random $(R)$ components for the c-axes $\langle 0001\rangle$ distribution. The points for each experiment are plotted every step of deformation $(\Delta \gamma=0.04)$.

data by Duval et al. (1983). We assumed that the equivalent stress at the first step of our numerical models $(\gamma=0.04)$ corresponds to the value observed for creep of isotropic polycrystals, at a similar strain rate and similar temperature (see Duval et al., 1983, Fig. 1).

\section{Results}

\subsection{Microstructure evolution}

Experiment 0, with neither GBM nor recovery, represents the highest strain rate and can be compared to the coldest polar ice that is deformed rapidly in simple shear (Fig. 2b). Deformation without recrystallization produced irregular elongated grains oriented parallel to the stretching direction at the end of the simulation (shear strain $\gamma=3$; Fig. 2b column I). In the absence of GBM, the average number of flynns (or grains) remained constant through the simulation (Table 1 ), but an intense network of subgrains is recognisable (Fig. 2 b). In this model deformation is localised in conjugate high-strain bands of strongly elongated grains, separated by low strain areas or microlithons (Fig. 2b column I) (Passchier and Trouw, 2005). During the simulation, subgrain misorientation develops progressively with increasing strain producing the local misorientation field shown in Fig. $2 \mathrm{~b}$ and indicating a general tendency to increase intergranular heterogeneities.

Experiments 1, 10 and 25 simulate systems with different degrees of $D R X$ and, hence, strain rates. In these cases, grains are still elongated parallel to the stretching direction and shape preferred orientations (SPO) are visible, despite the effect of DRX. However, grain-boundary migration decreases the extent of grain elongation, as $D R X$ produced larger and more equidimensional grains with straight to smooth boundaries. As a consequence, high-strain bands or low-strain regions are not obvious from the grain boundary network (Fig. 2d, e column I). The initial average grain size increased according to the amount of $D R X$ (Table 1 ).

\subsection{LPO evolution}

All simulations show a similar evolution of c-axis orientations, regardless of the amount of $D R X$. They start with randomly ori- 
Shear strain rate field
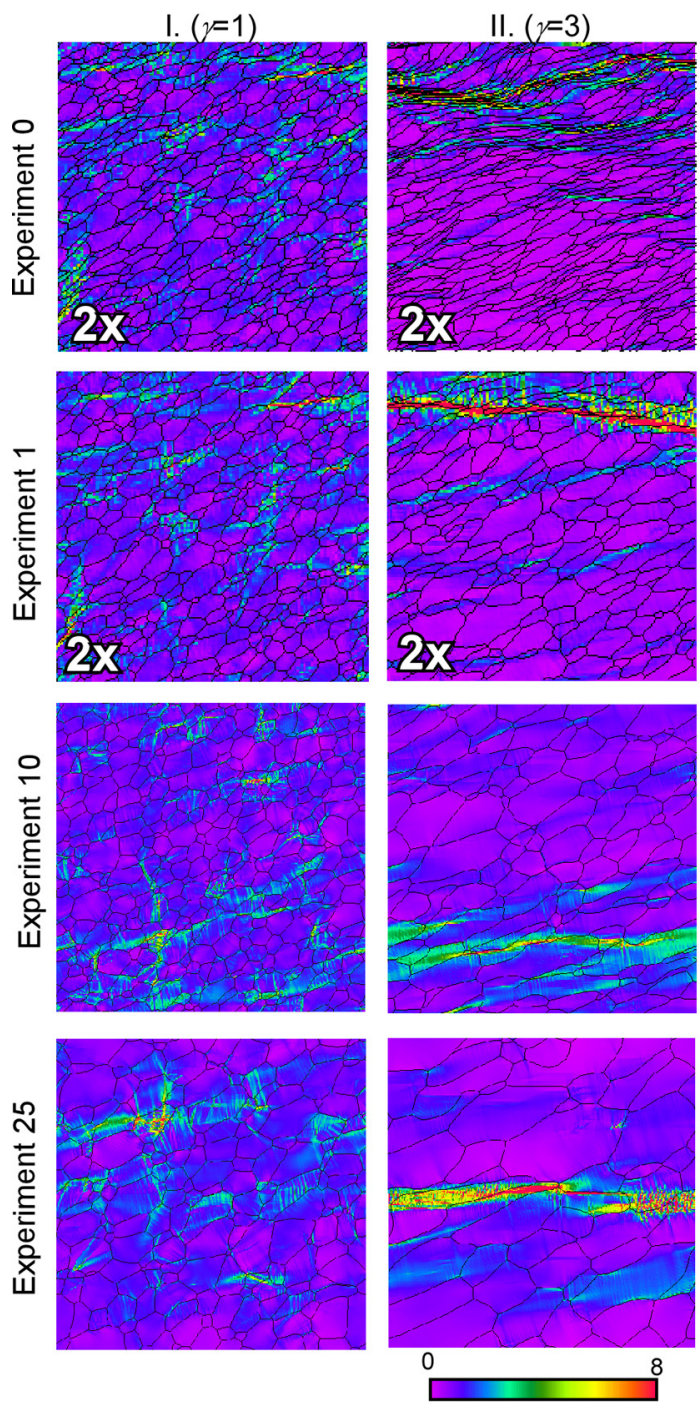

Passive marker grid
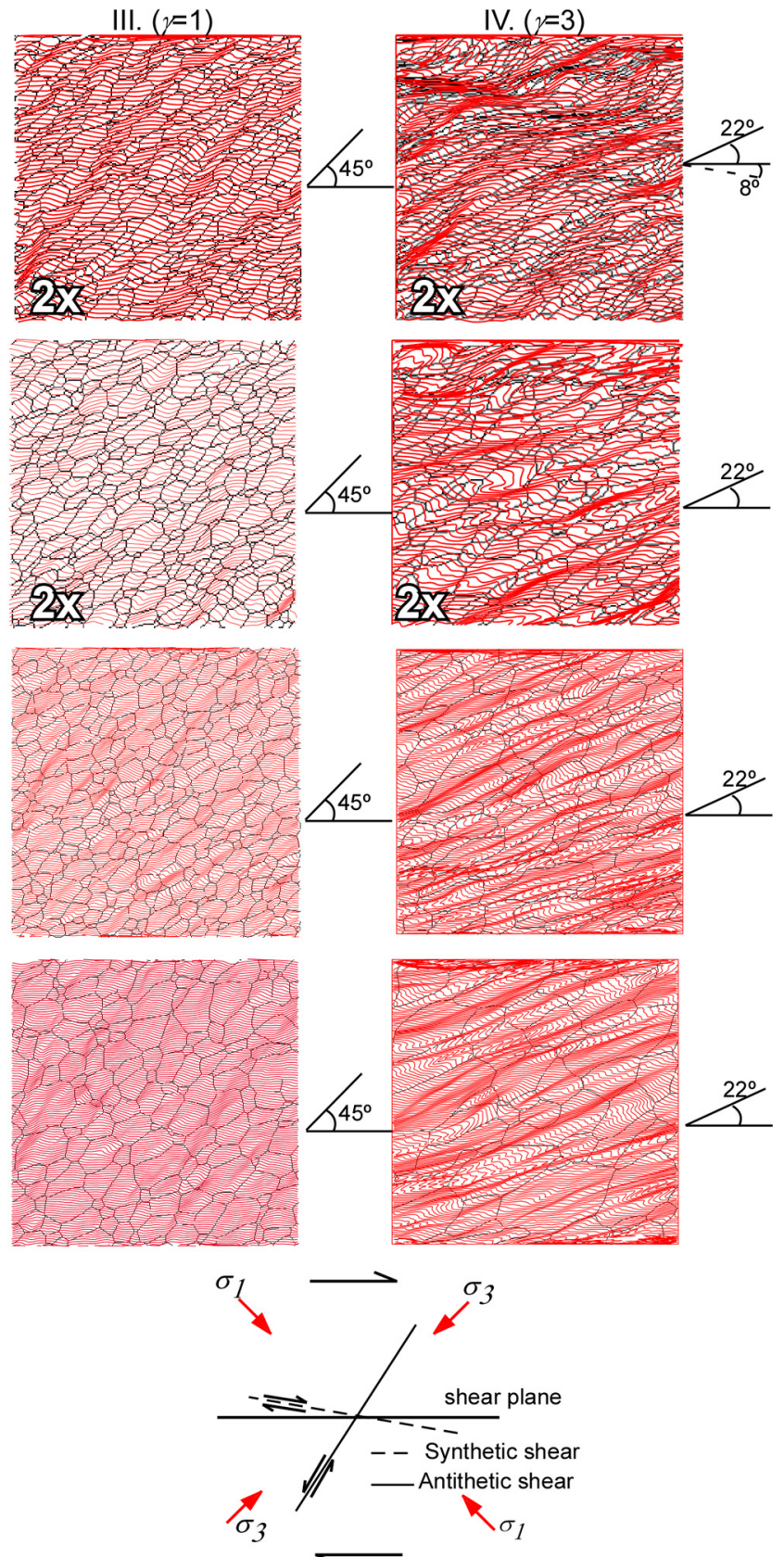

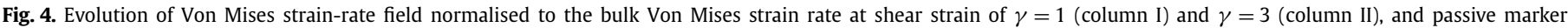

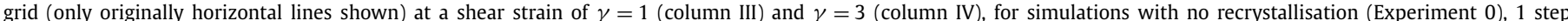

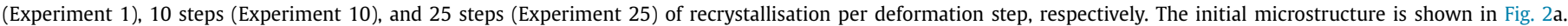
The grain boundary network is overlain in all images. Images for Experiments 0 and 1 have been enlarged $2 \times$, only showing a quarter of the model.

ented c-axes (Fig. 2a, columns II and III), and evolve to a c-axis orientation distribution approximately perpendicular to the shear plane (red and purple colours in Fig. 2b-e columns II and III). The preferred c-axis orientation is already clearly visible at a shear strain of $\gamma=1.5$ (Fig. 2b-e column III), where c-axes are oriented in an open cone which evolves further into a strong single maximum (Fig. 2b-e column III). The c-axes maximum progressively rotates towards the normal to the shear plane, but there is still an obliquity of about $20^{\circ}$ at $\gamma=3$ (Fig. 2b, e column III).

Whereas the effect of increasing GBM on the c-axes distribution is only minor, there is a distinct strengthening in alignment of the a- and b-axes (Fig. 2 column III). Without DRX, these be- come arranged in a broad girdle perpendicular to the c-axis maximum. When $D R X$ is active, the girdle is divided into a number of maxima, the strongest of which is in the direction of maximum elongation. This effect increases with increasing $D R X$ and is most pronounced for the a-axes distributions (Fig. 2d, e column III; movie Exp25_EBSD_ODF in supplementary material). The evolution of the fabric type during deformation is similar in all the simulations, with a rapid evolution towards a mixed girdle and point distribution, followed by a strengthening of the point maximum at high strain (Fig. 3). DRX increases the rate of this evolution, and furthermore strengthens the transient girdle component. 

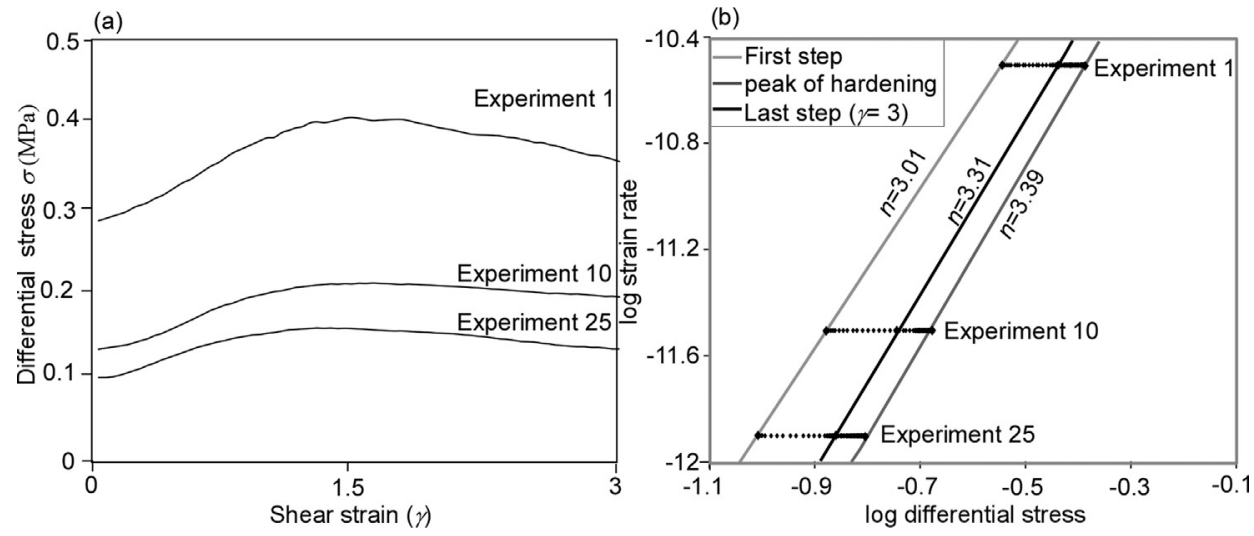

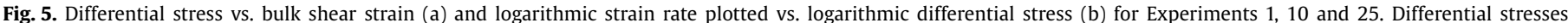
were normalised using the experimental values from Duval et al. (1983).

\subsection{Strain and strain rate localisation}

In all simulations, the strain-rate field becomes increasingly heterogeneous with progressive strain, and high strain-rate bands oriented at low angles to the horizontal shear plane (also called synthetic shear bands) develop (see columns I and II of Fig. 4). These shear bands are synthetic because they have the same sense of shear as the dextral bulk deformation and their intensity is about one order of magnitude higher than the bulk deformation (Fig. 4 column II). DRX reduces the number and intensity of shear bands (Experiment 10 and Experiment 25 in Fig. 4 columns I and II; movie Experiment25_grains_ugrid in supplementary material).

In all simulations, strain localisation is clearly recognisable by the deformed passive marker lines (Fig. 4 columns III and IV). At regions with high strain rate, the finite deformation patterns can be recognised from the microstructure (Experiments 0 and 1 in Fig. 4 columns III and IV). However, when DRX is intense enough it masks the strain rate heterogeneities, and passive markers are needed to visualise this strain localisation (Experiments 10 and 25 in Fig. 4 columns III and IV). Recrystallisation enables the grains to maintain an approximately elliptical shape that bears little or no relation with the heterogeneous finite-strain field (compare columns I in Fig. 2 and IV in Fig. 4). Contrary to the strain-rate field, the finite-strain distribution is dominated by oblique, antithetic shear bands that form at approximately $45^{\circ}$ to the shear plane at $\gamma=1$ (Fig. 4 column III), and progressively rotate to $22^{\circ}$ at the end of the simulations $(\gamma=3)$ (Fig. 4 column IV). The shear bands enclose lower-strain domains in which the originally horizontal grid lines are rotated clockwise and folded. In the absence of recrystallisation (Experiment 0), a second, synthetic set of shear bands develops approximately parallel to the shear plane, visible at the end of the simulations $(\gamma=3)$ (Experiment 0; Fig. 4 column IV). The intensity of strain localisation decreases and the spacing of shear bands increases with increasing recrystallisation.

\subsection{Stress evolution}

All simulations presented in this study follow a similar evolution characterised by an initial strain hardening, reaching a maximum of differential stress (normalised with respect to cf. Duval et al., 1983) which is followed by a softening at large strain (Fig. 5). The hardening and following softening stages are more pronounced in simulations performed at a high (Experiment 1) than at a low strain rate (Experiment 25) (Fig. 5a). Experiment 25 has the maximum value of stress at approximately shear strain of $\gamma=1$, while Experiment 1 requires a shear strain of $\gamma=1.5$ to reach the maximum value of stress (Fig. $5 \mathrm{a}$ ).
The evolution of the bulk stress exponent is observable in Fig. $5 \mathrm{~b}$, where the macroscopic stress exponent is calculated assuming that simulations performed at different strain rates, can be comparable at 3 different stages: initial, maximum value of normalised stress and final. The initial bulk stress exponent $n=3$ is coherent with imposed exponent for the individual slip systems. With progressive deformation the bulk stress exponent increases up to a maximum value of $n=3.4$ at the hardening peak, and decreases again to an $n=3.3$ at the end of the simulations.

\section{Discussion}

\subsection{Grain morphology and strain distribution}

The simulations show the strong, but not unexpected effect of recrystallisation on the grain shape and size evolution during deformation. In the absence of recrystallisation, the average aspect ratio $\left(R_{f}\right)$ of grains is about five at $\gamma=3$. This is about half of the finite strain ellipse at this strain $\left(R_{f} \approx 11\right)$. The difference arises from the strain heterogeneity that causes some grains to stretch more, but many less. Recrystallisation reduces $R_{f}$ at $\gamma=3$ to between 2.4 and 2.0 for Experiments 1 and 25, respectively. It should be noted that new HAGB's are not treated as such numerically in the recrystallisation routine. This would reduce the increase in grain shape and grain size by dividing up grains (Roessiger et al., 2011; Mathiesen et al., 2004). Depending on the orientation of the new grain boundaries, average elongation could be either strengthened or weakened. However, this would also add grain boundary energy to the system and it is to be expected that this would even further reduce the average elongation of grains.

The simulations with recrystallisation, applicable to slowly deforming polar ice, not only show that the average grain shape does not reflect the applied finite strain, but, more importantly, that the grain shapes completely mask the heterogeneity in strain rate and finite strain. It is, therefore, almost impossible to detect and quantify strain localisation from the grain morphology (Fig. 4, Experiments 10 and 25). This may be significant when interpreting the sequential layering of climate proxies in polar ice cores (e.g. NEEM, 2013). The simulations indicate that material points are affected by mechanical dispersion, as shown by paths of material points that deviate from the applied horizontal simple shear by distances in the order of a grain size at $\gamma=3$. These deviations would be much larger at the very high shear strains that are found in the lower part of ice sheets. An implication is that the ice stratigraphy may become distorted on the small scale. Although this is known to happen on the large scale (NEEM, 2013), it is difficult to assess this on the small scale. Indications for small-scale disturbances are provided by so-called 
“cloudy bands" using dark-field microscopy (Svensson et al., 2005; S.H. Faria et al., 2014), which from a depth below about $1700 \mathrm{~m}$ often show folds and disturbances (Jansen et al., 2016).

\subsection{Effects of $D R X$ on the evolution of lattice preferred orientation}

The results of the presented simulations indicate that the LPO evolve as expected in simple shear boundary conditions. This evolution is typically characterised by a strong single maximum almost perpendicular to the shear plane (Bouchez and Duval, 1982), as predicted by torsion experiments (Bouchez and Duval, 1982) and inferred from ice core data (S.H. Faria et al., 2014; Montagnat et al., 2014; Fitzpatrick et al., 2014). A strong LPO develops rapidly with increasing strain in all simulations, independent of whether $D R X$ is active or not, and regardless of the applied strain rate (Fig. 2 columns II and III). At lower shear strain $(\gamma=1.5$; Fig. 2 column III) the initial randomly oriented basal planes are quickly distributed into a preferred orientation at $\alpha=25.8^{\circ}$ with respect to the $y$-direction in all cases. Pole figures show that the single maximum is then rotated in the sense of shear up to approximately $\alpha=20^{\circ}$ at high strain $(\gamma=3$; Fig. 2 column III). Recrystallisation adds a minor girdle component to the LPO symmetry (Fig. 2c-e column III), which is more pronounced at low strain rates (Fig. 2d, e). This effect is clearly observable in our simulations (Fig. 3), where Experiments 10 and 25 develop a girdle component that forms at low shear strain (less than $\gamma=0.5$ ), despite the fact that all the simulations follow the same random to pointed LPO symmetry evolution.

Slight differences can be observed between our results and experimental, low strain simple shear tests (Bouchez and Duval, 1982; Kamb, 1972). These studies showed that the LPOs are sometimes accompanied by one or more secondary maxima rotated away in a direction reverse to the sense of shearing. Such bimodal or multiple maxima LPOs have been explained by grainboundary migration and/or preferential consumption of grains with unfavourable oriented lattices (Bouchez and Duval, 1982; Kizaki, 1969).

Our simulations demonstrate that LPO distributions are consistent with those described for quartz with an easy basal glide and a CRSS-ratio of basal vs. pyramidal and prismatic slip systems of three (Lister and Hobbs, 1980). This general LPO development is also similar to that described for ice from drill core observations and laboratory experiments (Bouchez and Duval, 1982). Our simulations, both with and without $D R X$, display a single c-axis maximum consistent with predominant slip activity along the basal plane. The preferred orientation of the basal planes is initially at a high, $\sim 60^{\circ}$ angle to the shear plane. When deformation continues, the LPO strengthens and the simple shear boundary conditions impose a rotation of basal planes, which produces a final preferred caxis orientation that tends to be perpendicular to the shear plane. This result is consistent with previous numerical simulations at low strains (e.g. Van der Veen and Whillans, 1994), where crystals are oriented in a single maximum fabric at $45^{\circ}$ with respect to the shear plane when $D R X$ is not active. However, these authors suggested that it is necessary to activate recrystallisation in order to develop a fabric almost perpendicular to the shear plane, as observed in nature at high strains. Our simulations show very similar results to the ones presented by Azuma (1994), where dextral simple shear simulations produce a single c-axes maximum that rotates clockwise towards almost perpendicular to the shear plane at high strains. Therefore, our results are consistent with the conclusion that it is not necessary to activate dynamic recrystallisation in order to develop the simple shear LPO as observed in nature (Azuma, 1994).

In general, the single maximum develops perpendicular to the direction of maximum finite shortening, which is initially $45^{\circ}$ to the shear plane in simple shear and subsequently rotates towards perpendicular to the shear plane. However, at the early stages, the maximum makes a larger angle than the expected $45^{\circ}$. This can be explained by the heterogeneity of strain (Fig. 4), where part of the strain is accommodated by steep antithetic shear bands. Zones between these shear bands rotate in a bookshelf manner. To compensate for the shear-plane parallel stretching that would result from this, a shortening component perpendicular to the shear bands develops. Most of the material thus experiences a maximum shortening component that is $<45^{\circ}$ inclined to the shear plane, as is reflected by the orientation of the c-axes maximum.

The LPOs obtained in the simulations are in accordance with the majority of ice drill core observations in Antarctica and Greenland (S.H. Faria et al., 2014). In deeper parts of the cores, where horizontal shear deformation is the main deformation component, most ice cores reveal an approximately vertical single maximum LPO (e.g. Herron and Langway, 1982; Thorsteinsson et al., 1997). Our results suggest that the LPO observed in these cores can be considered as being mainly deformation-induced, rather than significantly altered by recrystallisation. In the deepest part of some cores however, multiple maxima have been reported (Gow et al., 1997; Montagnat et al., 2014). Some aspects have to be considered in this context: (1) the ice is close to the pressure melting point close to the bedrock, (2) strain rates are generally highest in deepest parts and (3) grain sizes become very large, typically larger than available sample sizes from drill cores. The latter questions the relevance of distribution measurements. (1) and (2) are strongly connected in the sense that both do contribute to dynamic grain growth (H.G. Faria et al., 2014). Grain boundary mobility is significantly enhanced at high temperature and high strain rates enhance grain boundary migration. However, without the inclusion of grain boundary formation by polygonisation and nucleation this issue cannot yet be resolved here.

As expected, a-axes rotate towards the maximum elongation axis at low strain rates (Experiments 10 and 25) while c-axes rotate towards the direction of maximum finite shortening (Fig. $2 \mathrm{~d}$, e column III). This a-axis LPO is related to the increased activity of non-basal slip systems with increasing recrystallisation (Experiments 10 and 25 in Fig. 6). We suggest that basal slip can remain dominant in case of zero or low recrystallisation rates because permanent or long-lasting shear bands that accommodate most of the strain develop (Figs. 2 and 4). These shear bands consist of through-going bands of grains with favourably oriented basal planes. At higher recrystallisation rates, grain boundary migration destroys these bands necessitating deformation of grains with unfavourably oriented lattices in which non-basal slip is activated. Shear localisation remains important, but shear bands become ephemeral and vary in position over time. The enhancement of non-basal-slip activity by dynamic recrystallisation may then play an important role in deepest parts of the ice sheets, breaking down the strong single maxima LPO.

\subsection{Effects of $D R X$ on stress evolution}

The hardening and consecutive softening evolution followed for all simulations presented is more pronounced in models performed at high strain rate (Experiment 1), than in those deformed at low strain rate (Experiment 25) (Fig. 5a). This is in accordance with the standard concept of strain rate evolution with strain in ice (Treverrow et al., 2012) and other materials: dynamic recrystallisation inhibits hardening. The amount of $D R X$ has an influence on the strain hardening stage. The simulation performed at low strain rate requires a shear strain of $\gamma=1.5$ to reach the maximum value of stress (Experiment 1). When strain rate is lower the maximum value of stress is reached at approximately shear strain of $\gamma=1$ (Experiment 25) (Fig. 5a). 

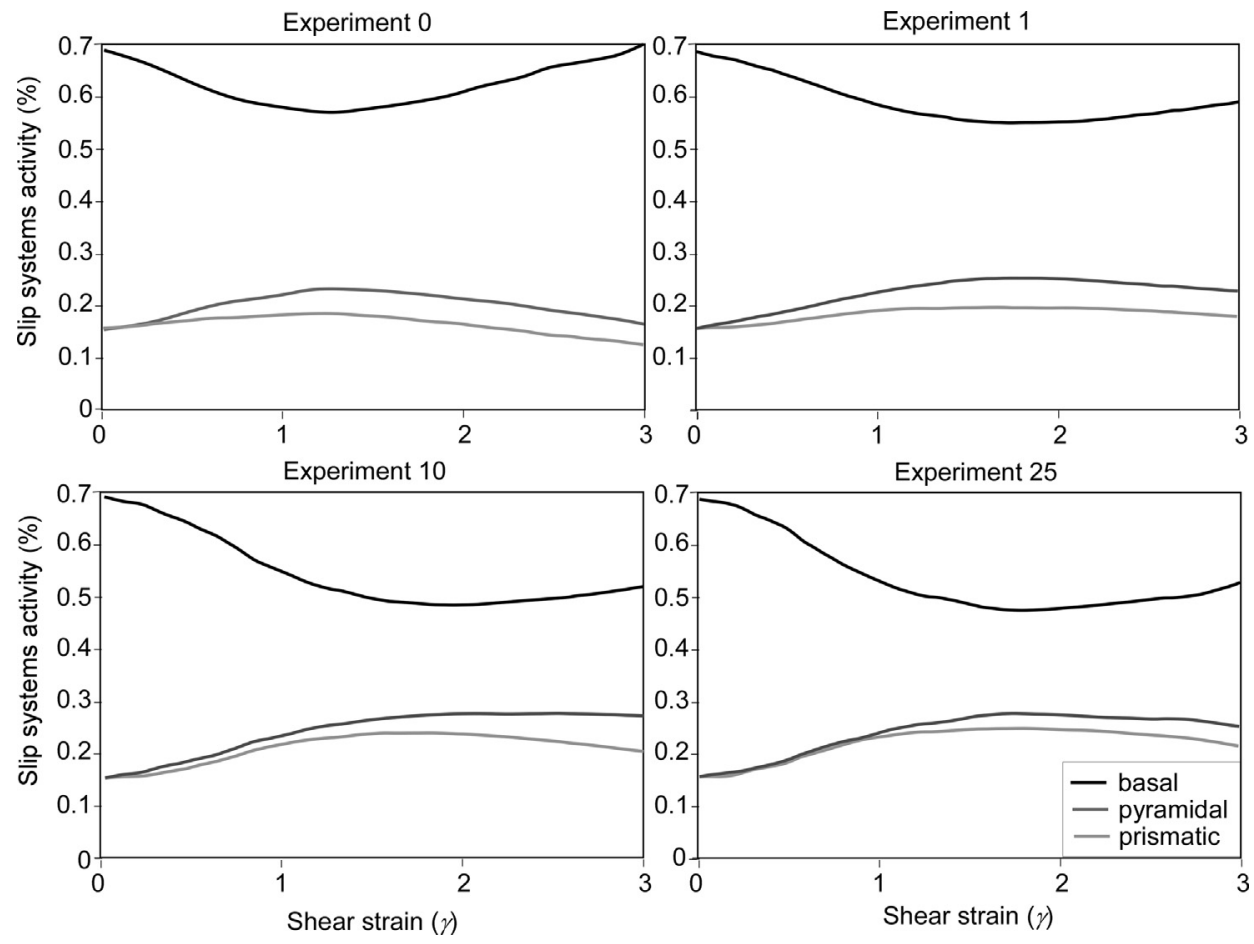

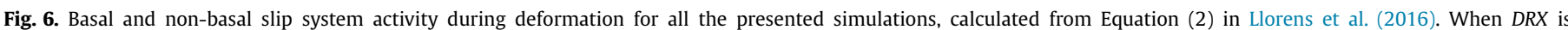
activated (Experiments 10 and 25) the activity of non-basal slip systems increases.

The increase of the bulk stress exponent shown in Fig. 5b can be explained by the $D R X$-dependent activation of the non-basal slip systems (Fig. 6), when the basal plane is oriented perpendicular to the maximum shortening direction (approximately at $45^{\circ}$ with respect to the shear plane). However, with increasing shear strain, basal planes tend to rotate towards the shear plane, at $45^{\circ}$ with respect to the tensile axis $\left(\sigma_{3}\right)$, resulting in softening as they become favourably oriented for basal slip. A similar effect can be observed during the development of a shape-preferred orientation (SPO) in a composite material: grains tend to elongate perpendicular to the maximum shortening direction, but at high shear strain some grains develop an elongation parallel to the shear plane, producing strain softening (Takeda and Griera, 2006).

\subsection{Recognising strain localisation in natural ice}

The macroscopic softening behaviour of ice-age ice has serious implications for ice core dating and paleoclimate record interpretation (Paterson, 1991). It has been suggested that this softening behaviour was produced by preferred orientations of the crystal lattice. However, investigations of glacial ice microstructures from the EDML deep ice core (Faria et al., 2009) suggest that dynamic grain boundary structures possibly formed by microshear, result in a reduction of the material strength. This was proposed to cause the softening behaviour of the ice-age ice, with small grain size ice deformed at relatively high temperature and moderate stress conditions (Faria et al., 2009). The accommodation of deformation by microshear reduces the stress concentration without modifying the bulk crystallographic texture evolution. Dislocation activity within grains produces microshear zones along grain boundary chains, splitting grains developing a subgrain boundary (Faria et al., 2009). Fig. 7 shows a qualitative comparison of our simulations with Antarctic ice samples from the EDML ice core (Faria et al., 2009). In our simulations, grains with zig-zag geometries and necking zones are indicators of localised high-strain bands, synchronous with grain coarsening caused by DRX (delimitated grains in red in Fig. 7c, e). Stepped geometries of grain boundaries par- allel to the shear plane (red arrows in Fig. $7 b-c$ ) are observable in local high strain bands (Fig. 7d-e). Therefore, we show how the presence of grains with zig-zag geometries and stepped grain boundaries can be used as indicators of strain localisation masked by grain boundary migration, features that are also observed in samples from the EDML ice core (Fig. 7a). The stepped geometries have been previously interpreted as precursors of microshearing. This interpretation was based on analogue experiments by Bons and Jessell (1999) using octachloropropane $\left(\mathrm{C}_{3} \mathrm{Cl}_{8}\right)$ at high homologous temperature. These authors observed that small bands of intense deformation did not produce an apparent grain size reduction or microstructural modification, but correlated with the development of stepped grain boundaries. Microshearing can combine grain boundary sliding (GBS) activity with dislocation motion in conventional creep, which is also termed Rachinger GBS (Langdon, 2006). The arrangement of misorientation structures in a stepped arrangement, as observed in our simulations may facilitate the onset of GBS so far only observed experimentally in ice (Goldsby and Kohlstedt, 2001). Our simulation results imply that microstructure analysis from certain ice core intervals would need to be revised in order to detect signs of masked strain localisation and viscous softening.

\section{Conclusions}

We presented novel full-field numerical simulations of ice $I h$ aggregates deformed in simple shear with and without recrystallisation. The simulations show that the ice crystal anisotropy is transferred to the polycrystal scale, where the distributions of both strain rate and finite strain are highly heterogeneous with the development of synthetic and antithetic shear bands. However, grain-boundary migration allows the grains to maintain an approximately elliptical shape, which masks the heterogeneous strain (rate) distribution. This makes it difficult to detect the presence of such strain heterogeneity in natural ice. However, masked shear localisation bands can be recognised from the presence of grains with zig-zag geometries and stepped boundaries. These features 
(a)

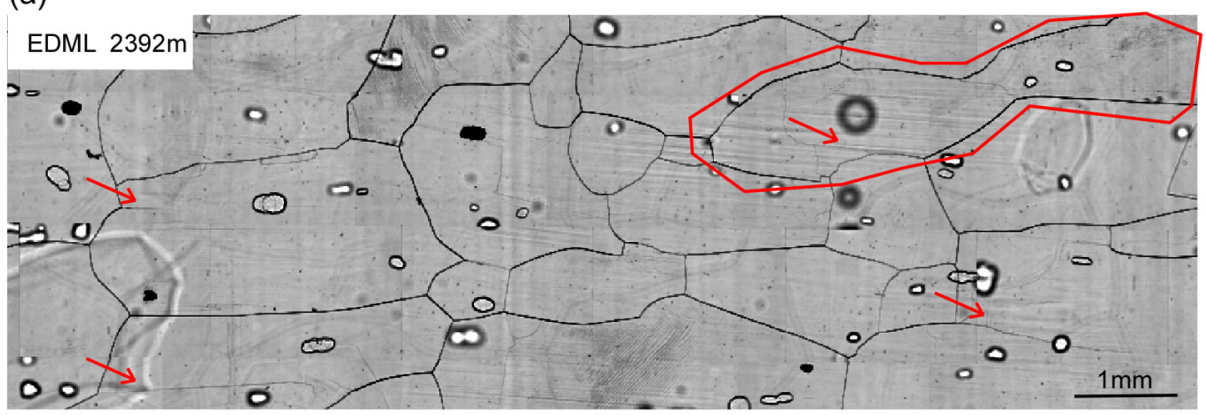

(b) Experiment 25

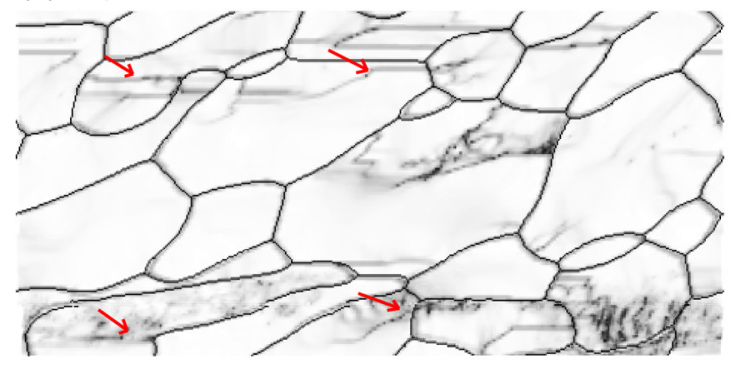

(d)

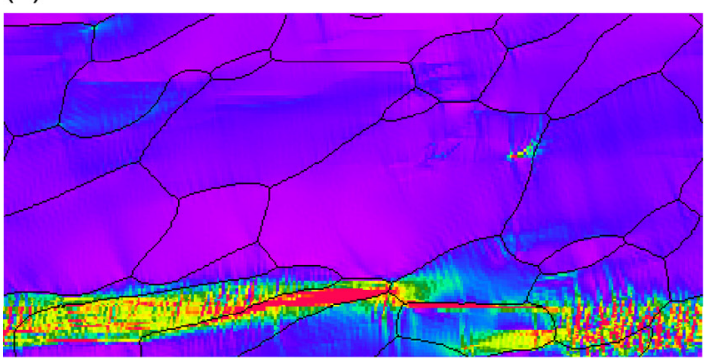

(c) Experiment 10

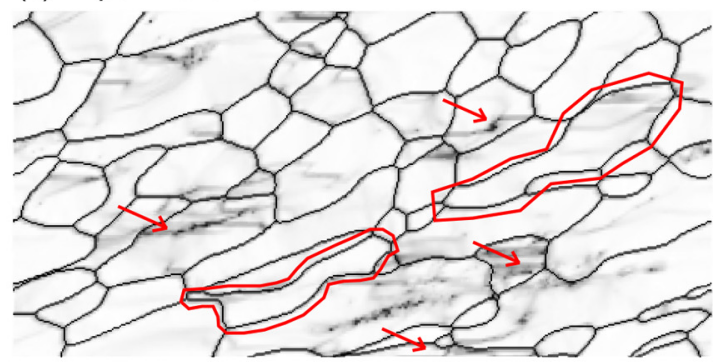

(e)

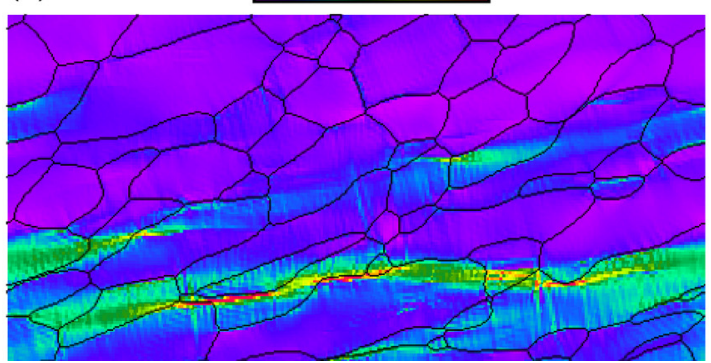

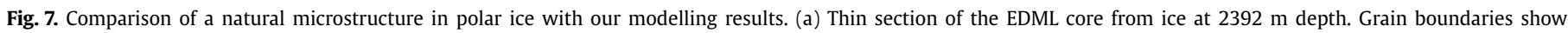

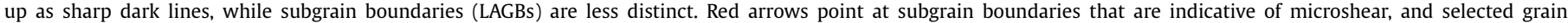

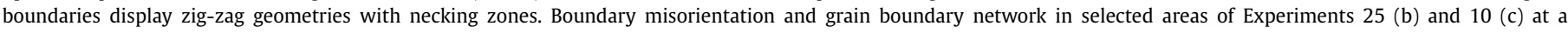

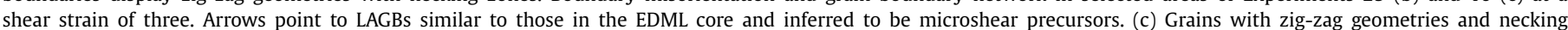

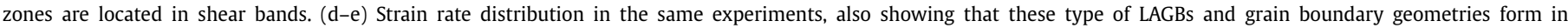
synthetic shear bands.

are observed in ice cores and can be considered as potential precursors of microshear in polar ice cores.

Although the amount of recrystallisation strongly affects the grain morphology, it has a much smaller effect on LPO development. In all cases a single c-axis maximum oriented approximately perpendicular to the maximum finite shortening direction develops. This maximum subsequently rotates towards the normal to the shear plane. The main impact of recrystallisation is the activation of non-basal slip, as required by the preferential alignment of basal planes initially normal to the maximum compression direction, which leads to a bulk hardening of the material at shear strain of $\gamma \approx 1-1.5$. The material subsequently softens again, as the basal planes rotate towards the shear plane and reach more favourable orientations for basal slip. As the amount of hardening depends on the amount of recrystallisation, and hence strain rate, the bulk stress exponent is higher (up to $\sim 3.4$ ) than that of the individual slip systems, which was set at three.

Our results indicate that the flow of ice may be much more heterogeneous than generally assumed, as neither the LPO nor the microstructure clearly reveals this heterogeneity. This has implications for the prediction of the rheology of natural ice, which may be weakened by the presence of shear bands. Even though these only occupy a small fraction of the material, they play a significant role in softening ice. Another implication of strain heterogeneity is the potential mixing or effective mechanical diffusion of fine particles and inclusions in the ice that carry climate signals.

\section{Acknowledgements}

We thank all the members of the ELLE development group, in particular Sandra Piazolo and Verity Borthwick for their contributions to the simulation code. The project was funded by the HGF (grant VH-NG-802). The Microdynamics of Ice (MicroDICE) research network, funded by the European Science Foundation, is acknowledged for funding research visits of MGL (short visit grants). We gratefully acknowledge the journal editor An Yin and anonymous reviewer, whose constructive reviews greatly improved the manuscript.

\section{Appendix A. Supplementary material}

Supplementary material related to this article can be found online at http://dx.doi.org/10.1016/j.epsl.2016.06.045. 


\section{References}

Alley, R., 1988. Fabrics in polar ice sheets: development and prediction. Science 240, 193-495.

Azuma, N., 1994. A flow law for anisotropic ice and its application to ice sheets. Earth Planet. Sci. Lett. 128 (3), 601-614.

Becker, J.K., Bons, P.D., Jessell, M.W., 2008. A new front-tracking method to model anisotropic grain and phase boundary motion in rocks. Comput. Geosci. 34 (3), 201-212.

Bons, P.D., Jessell, M.W., 1999. Micro-shear zones in experimentally deformed octachloropropane. J. Struct. Geol. 21, 323-334.

Bons, P.D., Koehn, D., Jessell, M.W., 2008. In: Bons, P., Koehn, D., Jessell, M. (Eds.), Microdynamic Simulation. In: Lecture Notes in Earth Sciences, vol. 106. Springer, Berlin, 405 pp.

Borthwick, V.E., Piazolo, S., Evans, L., Griera, A., Bons, P.D., 2013. What happens to deformed rocks after deformation? A refined model for recovery based on numerical simulations. Geol. Soc. (Lond.) Spec. Publ. 394 (1), 215-534.

Bouchez, J.L., Duval, P., 1982. The fabric of polycrystalline ice deformed in simple shear: experiments in torsion, natural deformation and geometrical interpretation. Texture Stress Microstruct. 5 (3), 171-190.

Budd, W.F., Jacka, H., 1989. A review of ice rheology for ice sheet modelling. Cold Reg. Sci. Technol. 16 (2), 107-144.

Castelnau, O., Duval, P., Lebensohn, R.A., Canova, G.R., 1996. Viscoplastic modeling of texture development in polycrystalline ice with a self-consistent approach: comparison with bound estimates. J. Geophys. Res., Solid Earth 101 (B6), 13851-13868.

Cuffey, K., Paterson, W.S.B., 2010. The Physics of Glaciers. Academic Press.

Duval, P., Ashby, M., Anderman, I., 1983. Rate controlling processes in the creep of polycrystalline ice. J. Phys. Chem. 87 (21), 4066-4074.

Duval, P., Castelnau, O., 1995. Dynamic recrystallization of ice in polar ice sheets. J. Phys. IV 5 (C3), C3-197.

Faria, H.G., Weikusat, I., Azuma, N., 2014. The microstructure of polar ice. Part II: state of the art. J. Struct. Geol. 61, 21-49.

Faria, S.H., Kipfstuhl, S., Azuma, N., Freitag, J., Hamann, I., Murshed, M.M., Kuhs, W.F., 2009. The multiscale structure of Antarctica. Part I: inland ice. Low Temp. Sci. 68, 39-59.

Faria, S.H., Freitag, J., Kipfstuhl, S., 2010. Polar ice structure and the integrity of ice-core paleoclimate records. Quat. Sci. Rev. 29, 338-351.

Faria, S.H., Weikusat, I., Azuma, N., 2014. The microstructure of polar ice. Part I: highlights from ice core research. J. Struct. Geol. 61, 2-20.

Fitzpatrick, J.J., Voight, D.E., Fegyveresi, J.M., Stevens, N.T., Spencer, M.K., Cole-Dai, J., Alley, R.B., Jardine, G.E., Cravens, E.D., Wilen, L.A., Fudge, T., McConnell, J.R. 2014. Physical properties of the WAIS Divide ice core. J. Glaciol. 60 (224), 1181-1198.

Goldsby, D.L., Kohlstedt, D.L., 2001. Superplastic deformation of ice: experimental observations. J. Geophys. Res., Solid Earth 106 (B6), 11017-11030.

Gow, A.J., Meese, D.A., Alley, R.B., Fitzpatrick, J.J., Anandakrishnan, S., Woods, G.A., Elder, B.C., 1997. Physical and structural properties of the Greenland Ice Sheet Project 2 ice core: a review. J. Geophys. Res. C 12, 26559-26576.

Griera, A., Bons, P.D., Jessell, M.W., Lebensohn, R.A., Evans, L., Gomez-Rivas, E., 2011. Strain localization and porphyroclast rotation. Geology 39, 275-278.

Griera, A., Llorens, M.-G., Gomez-Rivas, E., Bons, P.D., Jessell, M.W., Evans, L.A., Lebensohn, R.A., 2013. Numerical modelling of porphyroclast and porphyroblast rotation in anisotropic rocks. Tectonophysics 587, 4-29.

Herron, S.L., Langway, C.C., 1982. A comparison of ice fabrics and textures at Camp Century, Greenland and Byrd Station, Antarctica. Ann. Glaciol. 3, 118-124.

Jansen, D., Llorens, M.-G., Westhoff, J., Steinbach, F., Kipfstuhl, S., Bons, P.D., Griera, A., Weikusat, I., 2016. Small-scale disturbances in the stratigraphy of the NEEM ice core: observations and numerical model simulations. Cryosphere 10 (1), 359-370.

Kamb, W.B., 1972. Experimental recrystallization of ice under stress. In: Heard, H.C., Borg, I.Y., Carter, N.L., Raleigh, C.B. (Eds.), Flow and Fracture of Rocks. In: Geophysical Monograph, vol. 16. American Geophysical Union, Washington, DC, pp. 211-241.

Kipfstuhl, S., Hamann, I., Lambrecht, A., Freitag, J., Faria, S.H., Grigoriev, D., Azuma, N., 2006. Microstructure mapping: a new method for imaging deformationinduced microstructural features of ice on the grain scale. J. Glaciol. 52 (178), 398-406.
Kizaki, K., 1969. Ice-fabric study of the Mawson region, East Antarctica. J. Glaciol. 8 (53), 253-276.

Langdon, T.G., 2006. Grain boundary sliding revisited: developments in sliding over four decades. J. Mater. Sci. 41 (3), 597-609.

Lebensohn, R.A., 2001. N-site modelling of a 3D viscoplastic polycrystal using fast Fourier transform. Acta Mater. 49 (14), 2723-2737.

Lebensohn, R.A., Brenner, R., Castelnau, O., Rollett, A.D., 2008. Orientation imagebased micromechanical modelling of subgrain texture evolution in polycrystalline copper. Acta Mater. 56 (15), 3914-3926.

Lister, G.S., Hobbs, B.E., 1980. The simulation of fabric development during plastic deformation and its application to quartzite: the influence of deformation history. J. Struct. Geol. 2 (3), 355-370.

Llorens, M.-G., Bons, P.D., Griera, A., Gomez-Rivas, E., Evans, L.A., 2013a. Single layer folding in simple shear. J. Struct. Geol. 50, 209-220.

Llorens, M.-G., Bons, P.D., Griera, A., Gomez-Rivas, E., 2013b. When do folds unfold during progressive shear? Geology 41, 563-566.

Llorens, M.-G., Griera, A., Bons, P.D., Roessiger, J., Lebensohn, R., Evans, L., Weikusat I., 2016. Dynamic recrystallisation of ice aggregates during viscoplastic deformation: a numerical approach. J. Glaciol. 62, 359-377. http://dx.doi.org/ 10.1017/jog.2016.28.

Mainprice, D., Hielscher, R., Schaeben, H., 2011. Calculating anisotropic physical properties from texture data using the MTEX open source package. Geol. Soc. (Lond.) Spec. Publ. 360 (1), 175-192. http://dx.doi.org/10.1144/SP360.10.

Mathiesen, J., Ferkinghoff-Borg, J., Jensen, M.H., Levinsen, M., Olesen, P., Dahl-Jensen, D., Svensson, A., 2004. Dynamics of crystal formation in the Greenland NorthGRIP ice core. J. Glaciol. 50, 392-398.

Montagnat, M., Azuma, N., Dahl-Jensen, D., Eichler, J., Fujita, S., Gillet-Chaulet, F., Kipfstuhl, S., Samyn, D., Svensson, A., Weikusat, I., 2014. Fabric along the NEEM ice core, Greenland, and its comparison with GRIP and NGRIP ice cores. Cryosphere 8, 1129-1138.

Montagnat, M., Chauve, T., Barou, F., Tommasi, A., Beausir, B., Fressengeas, C., 2015 Analysis of dynamic recrystallization of ice from EBSD orientation mapping. Earth Sci. Front. 3, 81.

NEEM Community Members, 2013. Eemian interglacial reconstructed from a Greenland folded ice core. Nature 493 (7433), 489-494. http://dx.doi.org/ 10.1038/nature11789.

Passchier, C.W., Trouw, R.A.J., 2005. Microtectonics. Deformation Mechanisms, vol. 2. Springer, Berlin. 1996

Paterson, W.S.B., 1991. Why ice-age ice is sometimes "soft". Cold Reg. Sci. Technol. 20 (1), 75-98.

Piazolo, S., Bons, P.D., Jessell, M.W., Evans, L., Passchier, C.W., 2002. Dominance of microstructural processes and their effect on microstructural development: insights from numerical modelling of dynamic recrystallization. Geol. Soc. (Lond.) Spec. Publ. 200 (1), 149-170.

Roessiger, J., Bons, P.D., Griera, A., Jessell, M.W., Evans, L., Montagnat, M., Kipfstuhl, S., Faria, S.H., Weikusat, I., 2011. Competition between grain growth and grain size reduction in polar ice. J. Glaciol. 57 (205), 942-948.

Svensson, A., Nielsen, S.W., Kipfstuhl, S., Johnsen, S.J., Steffensen, J.P., Bigler, M., Ruth, U., Röthlisberger, R., 2005. Visual stratigraphy of the North Greenland Ice Core Project (NorthGRIP) ice core during the last glacial period. J. Geophys. Res., Atmos. 110 (D2), 1984-2012.

Takeda, Y.T., Griera, A., 2006. Rheological and kinematical responses to flow of twophase rocks. Tectonophysics 427 (1), 95-113.

Thorsteinsson, T., Kipfstuhl, J., Miller, H., 1997. Textures and fabrics in the GRIP ice core. J. Geophys. Res. 102, 26583-26599.

Treverrow, A., Budd, W.F., Jacka, T.H., Warner, R.C., 2012. The tertiary creep of polycrystalline ice: experimental evidence for stress-dependent levels of strain-rate enhancement. J. Glaciol. 58, 301-314.

Van der Veen, C.J., Whillans, I.M., 1994. Development of fabric in ice. Cold Reg. Sci. Technol. 22 (2), 171-195.

Vollmer, F.W., 1990. An application of eigenvalue methods to structural domain analysis. Geol. Soc. Am. Bull. 102 (6), 786-791.

Weikusat, I., Kipfstuhl, S., Azuma, N., Faria, S.H., Miyamoto, A., Hondoh, T., 2009. Deformation Microstructures in an Antarctic Ice Core (EDML) and in experimentally deformed. In: Artificial Ice. Physics of Ice Core Records II. Low Temp. Sci. 68 (suppl.), 115-123.

Woodcock, N.H., 1977. Specification of fabric shapes using an eigenvalues method. Geol. Soc. Am. Bull. 88 (9), 1231-1236. 